\title{
AN ASYMPTOTIC SOLUTION OF AN INTEGRO-DIFFERENTIAL EQUATION ARISING IN MAGNETIC COUPLING THROUGH THIN SHIELD WALLS
}

\author{
G. F. FITZ-GERALD ${ }^{1}$ and N. A. McDONALD ${ }^{2}$
}

(Received 7 February 1988; revised 22 August 1988)

\begin{abstract}
An equation which has arisen in a study of the magnetic coupling through a small rectangular aperture of dimension $A \times B$ in a thin shield wall is discussed. The magnetic polarisability of such an aperture in a conducting wall of zero thickness is known to be expressible as $R_{H} A^{3}$, in which $R_{H}$ is dimensionless and is a function of the aspect ratio $\alpha \equiv B / A$. An asymptotic solution procedure of a certain variational formulation of this problem is described in the limiting case of large aspect ratio $\alpha$. Explicit analytical formulae for the leading terms in the expansion are given. These analytical results justify the purely numerical procedures used previously to obtain approximate solutions of this formulation of the problem.
\end{abstract}

\section{Introduction}

This paper is concerned with a certain variational formulation that arose in a study of the electromagnetic field penetration through small apertures in thin metallic shield walls. An integro-differential equation for the "shape" function $\rho$ that minimises this variational statement is given, and an asymptotic solution for the "shape" function and the polarisability coefficient $R_{H}$ is obtained for large values of $\alpha$.

Most work on this topic of small apertures in electromagnetics is based on the work of [2] which showed that the field radiating into one region from another via an aperture which is small compared with the wavelength of the exciting field, is the same as that resulting from a combination of electric and magnetic

\footnotetext{
${ }^{1}$ Department of Mathematics, RMIT, Melbourne, Vic. Australia.

${ }^{2}$ Department of Communication and Electronic Engineering, RMIT, Melbourne, Vic. Australia. (C) Copyright Australian Mathematical Society 1989, Serial-fee code 0334-2700/89
} 
dipole sources, the former associated with the normal electric field at the aperture location and the latter associated with the tangential magnetic field. The relationship between the exciting field (electric or magnetic component) and the resulting dipole moment is referred to as the polarisability (electric or magnetic) of the aperture. More specifically, the dipole moments are calcuated from the excitation fields which exist prior to opening the aperture (and which are therefore independent of the aperture size and shape) and the polarisabilities, which are functions of the aperture size and shape but independent of the excitation. In this way the problem is partitioned into excitation and aperture components.

It has been established $[2,3]$ that each aperture polarisability has the dimensions of (length) ${ }^{3}$. Accordingly, for a given shape, the polarisability can be expressed as a dimensionless function of the ratio of width to length (here referred to as the polarisability coefficient) multiplied by the cube of a characteristic dimension. For example, the magnetic polarisability of a rectangular aperture of dimension $A \times B$ is expressible as $R_{H} A^{3}$ in which $R_{H}$ is a dimensionless function of the aspect ratio $B / A$.

In the particular case of the magnetic polarisability of a square aperture, it is recognised in the literature (see, for example, [10]) that there is a spread of approximately $4 \%$ in the values obtained by different authors for the magnetic polarisability coefficient $R_{H}$. Because these values have arisen as numerical estimates from distinctly different methods of solution applied to completely different formulations of the problem, it is not possible to compare results at any intermediate stage of the various solution procedures.

The method adopted in [5], for example, is entirely numerical and based on a finite element formulation. Associated with the mathematical statement of the problem in such rectangular regions are the inherent difficulties faced by any purely numerical procedure; in particular, the known singular behaviour of the solution occurring in the vicinity of the corners of such regions.

The method adopted by $[8,9]$ to calculate the magnetic polarisability of rectangular apertures uses a variational formulation and a numerical Ritz procedure based on a sum of twelve $T E_{n 0}$ waveguide mode trial functions. The benefit of using waveguide mode functions was that any wall thickness could be accommodated by that approach. However, there is considerable interest in numerical values for the special case of zero, or negligibly small, wall thickness. For this purpose, the more general variational formulation $[8,9]$ for $R_{H}$ has been recast into a more attractive form for this special case; see (2.1). This has been achieved by replacing the truncated modal sum used in $[8,9]$ by a general "shape" function $\rho$ that is to be chosen to minimise the relevant expression for the polarisability coefficient $R_{H}$ for each value of the aspect ratio $\alpha$. 
Physically, $\rho$ is proportional to the electric field strength across the aperture and because of the variational formulation, its absolute magnitude is irrelevant. Also in the derivation of (2.1), the aperture length $A$ is normalised to unity, so that the electric field $\rho(a)$, which is dependent on the position $a$ along the aperture, exists only in the interval $[-1 / 2,1 / 2]$. From arguments based on physical considerations, the "shape" function $\rho$ is assumed to be symmetric on the interval $[-1 / 2,1 / 2]$, to vanish at $\pm 1 / 2$ and to be suitably differentiable on $(-1 / 2,1 / 2)$. With no loss of generality this shape function $\rho$ can be normalised so that $\int_{0}^{\frac{1}{2}} \rho(a) d a=1$.

It is worth noting that for smooth regions, such as a circle and an ellipse, the numerical techniques of $[8,9]$ and [5] yield nearly identical results. Moreover, these results are in excellent agreement with the known exact solution, see for example [2] and [4].

In this paper the numerical results obtained by $[8,9]$ using a variational formulation for rectangular apertures in thin shield walls are shown analytically to be correct to better than four figure accuracy in the limiting case of the aspect ratio $\alpha$ large compared with unity.

\section{The integro-differential equation}

In $[8,9]$, a Calculus of Variations procedure was used to obtain an expression for the dimensionless polarisability coefficient $R_{H}$ with a sum of $T E_{n 0}$ mode fields as the trial functions. The replacement of the approximating modal sum by the "shape" function $\rho$ in that work leads to the following expression for the dimensionless polarisability coefficient $R_{H}$.

$$
\frac{1}{R_{H}}=\frac{8}{\pi^{2}} \int_{0}^{\infty} \int_{0}^{\infty} \frac{u^{2} \sin ^{2}(v / 2)}{v^{2} \sqrt{v^{2}+\alpha^{2} u^{2}}}\left[\int_{0}^{\frac{1}{2}} \rho(a) \cos a u d a\right]^{2} d u d v .
$$

The objective of the present project is to find an analytic solution of the variational formulation (2.1). The two main reasons for desiring such an analytic solution are:

- to confirm the numerical results obtained earlier;

- to provide a basis for the resolution of the discrepancies between the values obtained by other workers using different formulations.

A linear change of both variables, followed by a further linear change of the resulting inner integration variable and use of the result

$$
\int_{0}^{\infty} \frac{\sin ^{2} y t}{t^{2} \sqrt{1+t^{2}}} d t=y\left[K_{1}(2 y)-\frac{1}{2 y}-K i_{1}(2 y)+\frac{\pi}{2}\right]
$$


where $K_{1}$ is the modified Bessel function of the first kind of order one, and $K i_{1}$ is the first integral of $K_{0}$, the Bessel function of the third kind of order zero, together with the assumed evenness of $\rho$ yields the result

$$
\begin{aligned}
\frac{1}{R_{H}}= & \frac{4}{\pi^{2} \alpha^{3}} \int_{0}^{\infty}\left(\frac{1}{2} \pi w-\frac{1}{2}\right)\left[\int_{-\frac{1}{2}}^{\frac{1}{2}} \rho(a) \cos \left(\frac{2 w a}{\alpha}\right) d a\right]^{2} d w \\
& +\frac{4}{\pi^{2} \alpha^{3}} \int_{0}^{\infty} F(w) \int_{-\frac{1}{2}}^{\frac{1}{2}} \rho(a) \cos \left(\frac{2 w a}{\alpha}\right) d a d w
\end{aligned}
$$

where $F(w) \equiv w\left(K_{1}(2 w)-K i_{1}(2 w)\right) \int_{-\frac{1}{2}}^{\frac{1}{2}} \rho(b) \cos (2 w b / \alpha) d b$ (see [6] for details here and throughout this section).

Extensive use of the tables of [7] leads to the following three identities needed in the subsequent simplification of (2.2) for the polarisability coefficient $R_{H}$ :

$$
\begin{gathered}
\int_{0}^{\infty} w \cos \frac{2 w(a \mp b)}{\alpha} K_{1}(2 w) d w=\frac{\pi}{8}\left(1+\left(\frac{a \mp b}{\alpha}\right)^{2}\right)^{-\frac{3}{2}} \\
\int_{0}^{\infty} w \cos \frac{2 w(a \mp b)}{\alpha} K i_{1}(2 w) d w \\
=\frac{\pi}{8}\left[\left(1+\left(\frac{a \mp b}{\alpha}\right)^{2}\right)^{-\frac{3}{2}}+\left(\frac{a \mp b}{\alpha}\right)^{-2}\left(1+\left(\frac{a \mp b}{\alpha}\right)^{2}\right)^{-\frac{1}{2}}-\left(\frac{a \mp b}{\alpha}\right)^{-2}\right],
\end{gathered}
$$

and

$$
\begin{aligned}
\int_{-\frac{1}{2}}^{\frac{1}{2}} \rho^{\prime}(a) & d a \int_{-\frac{1}{2}}^{\frac{1}{2}} \rho^{\prime}(b) d b \int_{0}^{\infty} \frac{\sin (2 w a / \alpha) \sin (2 w b / \alpha)}{w^{2}} d w \\
= & \frac{2 \pi}{\alpha} \int_{-\frac{1}{2}}^{\frac{1}{2}} \rho^{2}(a) d a .
\end{aligned}
$$

Further extensive use of the tables of $[7]$ together with the above three idenities and the assumed properties of the function $\rho$ yields the following simplified form for $R_{H}$.

$$
\begin{aligned}
\frac{1}{R_{H}}= & \int_{-\frac{1}{2}}^{\frac{1}{2}} \rho^{\prime}(a) d a \int_{-\frac{1}{2}}^{\frac{1}{2}} \rho^{\prime}(b) g(a, b) d b \\
& -\frac{1}{\pi \alpha^{2}} \int_{-\frac{1}{2}}^{\frac{1}{2}} \rho^{2}(a) d a+\int_{-\frac{1}{2}}^{\frac{1}{2}} \rho(a) d a \int_{-\frac{1}{2}}^{\frac{1}{2}} f(a, b) \rho(b) d b
\end{aligned}
$$


where the kernel functions $f$ and $g$ are defined by

$$
\begin{aligned}
f(a, b) & \equiv \frac{1}{2 \pi \alpha(a+b)^{2}}\left(1-\left(1+\left(\frac{a+b}{\alpha}\right)^{2}\right)^{-\frac{1}{2}}\right) \\
& =\frac{1}{2 \pi \alpha^{3}}\left(\sqrt{1+\left(\frac{a+b}{\alpha}\right)^{2}}\left(1+\sqrt{1+\left(\frac{a+b}{\alpha}\right)^{2}}\right)\right)^{-1},
\end{aligned}
$$

and $g(a, b) \equiv \frac{1}{8 \pi \alpha} \log \left(\frac{a+b}{a-b}\right)^{2}$.

Including the constraint imposed on $\rho$ by virtue of the normalisation requirement with the use of a Lagrange multiplier $\lambda$, and rearranging this last expression for $R_{H}$ into a more symmetric form leads to the expression

$$
\begin{aligned}
\frac{1}{R_{H}}=\int_{-\frac{1}{2}}^{\frac{1}{2}} \int_{-\frac{1}{2}}^{\frac{1}{2}}\left[\rho(a) \rho(b) f(a, b)+\rho^{\prime}(a) \rho^{\prime}(b) g(a, b)\right. \\
\\
\left.\quad-\frac{1}{2 \pi \alpha^{2}}\left(\rho^{2}(a)+\rho^{2}(b)\right)+\lambda(\rho(a) \rho(b)-4)\right] d a d b .
\end{aligned}
$$

Formal application of the standard argument of the Calculus of Variations (that is, replacing $\rho$ by $\rho+\varepsilon \eta$ with $\eta( \pm 1 / 2)=0$ and demanding that the coefficient of the term multiplying $\varepsilon$ vanishes) yields for stationarity of $R_{H}^{-1}$ the equation

$$
\begin{aligned}
& \int_{-\frac{1}{2}}^{\frac{1}{2}} \int_{-\frac{1}{2}}^{\frac{1}{2}}\left[(\rho(a) \eta(b)+\rho(b) \eta(a)) f(a, b)+\left(\rho^{\prime}(a) \eta^{\prime}(b)+\rho^{\prime}(b) \eta^{\prime}(a)\right) g(a, b)\right. \\
& \left.\quad-\frac{1}{2 \pi \alpha^{2}}(2 \rho(a) \eta(a)+2 \rho(b) \eta(b))+\lambda(\rho(a) \eta(b)+\rho(b) \eta(a))\right] d a d b=0 .
\end{aligned}
$$

Performing one integration by parts to remove the terms involving $\eta^{\prime}$ (but avoiding the introduction of terms in $\rho^{\prime \prime}$ because of the order of this function's singular behaviour near to $\pm 1 / 2$ ) and using simple symmetry properties of the introduced functions $f$ and $g$ yields as the requirement of stationarity of $R_{H}^{-1}$ the condition

$$
\int_{-\frac{1}{2}}^{\frac{1}{2}}\left[f_{-\frac{1}{2}}^{\frac{1}{2}}\left(\rho(a) f(a, b)-\rho^{\prime}(a) g_{b}(a, b)-\frac{1}{\pi \alpha^{2}} \rho(b)+\lambda \rho(a)\right) d a\right] \eta(b) d b=0 .
$$

The standard argument of the Calculus of Variations and use of the integral constraint imposed on the "shape" function $\rho$ then yields the result

$$
f_{-\frac{1}{2}}^{\frac{1}{2}}\left(\rho(a) f(a, b)-\rho^{\prime}(a) g_{b}(a, b)\right) d a-\frac{1}{\pi \alpha^{2}} \rho(b)+2 \lambda=0, \quad \forall b \in(-1 / 2,1 / 2) .
$$

The value of the Lagrange multiplier $\lambda$ is obtained by formally integrating (2.7) with respect to $b$ from $-1 / 2$ to $1 / 2$. 
Substitution of this value of $\lambda$ into the above equation and use of the definitions of $f$ and $g$ yields as the governing equation for the "shape" function $\rho$

$$
\begin{aligned}
& f_{-\frac{1}{2}}^{\frac{1}{2}} \rho^{\prime}(a) \frac{a}{a^{2}-b^{2}} d a \\
& \quad=\frac{4}{\alpha}-\frac{2}{\alpha} \rho(b)-M+\frac{1}{\alpha^{2}} \int_{-\frac{1}{2}}^{\frac{1}{2}} \frac{\rho(a)}{\sqrt{1+\left(\frac{a+b}{\alpha}\right)^{2}}\left(1+\sqrt{1+\left(\frac{a+b}{\alpha}\right)^{2}}\right)} d a
\end{aligned}
$$

where the value of $M$ is given by

$$
M=\int_{-\frac{1}{2}}^{\frac{1}{2}}\left[\sqrt{1+\left(\frac{\frac{1}{2}-a}{\alpha}\right)^{2}} /\left(\frac{1}{2}-a\right)+\sqrt{1+\left(\frac{\frac{1}{2}+a}{\alpha}\right)^{2}} /\left(\frac{1}{2}-a\right)\right] \rho(a) d a .
$$

The above equation (2.8) is a singular, Fredholm, integro-differential equation of the second kind for the "shape" function $\rho$. The stationary value $\left(R_{H}^{-1}\right)_{\text {stat }}$ of $R_{H}^{-1}$, as given by (2.4), can be simplified using the above integro-differential equation (2.8) to yield

$$
\left(R_{H}^{-1}\right)_{\mathrm{stat}}=\frac{\alpha M-4}{\pi \alpha^{2}} .
$$

\section{Asymptotic solution for large $\alpha$}

The limiting case $\alpha \gg 1$ is now considered and the asymptotic solution is sought using a standard regular perturbation expansion procedure. That is, the "shape" function $\rho$ is assumed to be expressible in the form $\rho(a)=$ $\rho^{(0)}(a)+\alpha^{-1} \rho^{(1)}(a)+\alpha^{-2} \rho^{(2)}(a)+\cdots$, whilst each term of the above integrodifferential-equation (2.8) is expanded in a power series of inverse powers of $\alpha$. Upon equating like powers of $\alpha^{-1}$ that then appear on either side of (2.8), the following sequence of problems for the first three terms $\rho^{(0)}, \rho^{(1)}$ and $\rho^{(2)}$ is obtained:

$$
\begin{gathered}
f_{-\frac{1}{2}}^{\frac{1}{2}} \rho^{(0)^{\prime}}(a) a /\left(a^{2}-b^{2}\right) d a=-M^{(0)} \\
f_{-\frac{1}{2}}^{\frac{1}{2}} \rho^{(1)^{\prime}}(a) a /\left(a^{2}-b^{2}\right) d a=4-2 \rho^{(0)}(b)-M^{(1)}
\end{gathered}
$$

and

$$
f_{-\frac{1}{2}}^{\frac{1}{2}} \rho^{(2)^{\prime}}(a) a /\left(a^{2}-b^{2}\right) d a=-2 \rho^{(1)}(b)+1 / 2 \int_{-\frac{1}{2}}^{\frac{1}{2}} \rho^{(0)}(a) d a-M^{(2)},
$$


where the constants $M^{(0)}, M^{(1)}$ and $M^{(2)}$ are defined by

$$
\begin{aligned}
& M^{(0)}=\int_{-\frac{1}{2}}^{\frac{1}{2}} \rho^{(0)}(a) /\left(\frac{1}{4}-a^{2}\right) d a, \\
& M^{(1)}=\int_{-\frac{1}{2}}^{\frac{1}{2}} \rho^{(1)}(a) /\left(\frac{1}{4}-a^{2}\right) d a,
\end{aligned}
$$

and

$$
M^{(2)}=\int_{-\frac{1}{2}}^{\frac{1}{2}} \rho^{(2)}(a) /\left(\frac{1}{4}-a^{2}\right) d a+1 / 2 \int_{-\frac{1}{2}}^{\frac{1}{2}} \rho^{(0)}(a) d a .
$$

The integral constraint condition arising from the normalising of the "shape" function $\rho$ further requires that $\int_{-\frac{1}{2}}^{\frac{1}{2}} \rho^{(0)}(a) d a=2$, and $\int_{-\frac{1}{2}}^{\frac{1}{2}} \rho^{(n)}(a) d a=0$, for $n=1,2$. Clearly, similar equations to equations (3.1) to (3.6) can be obtained for all of the higher order terms.

In terms of the values $M^{(0)}, M^{(1)}$ and $M^{(2)}$, the leading terms that represent the stationary value of $R_{H}^{-1}$ (also expanded in inverse powers of $\alpha$ ) are given by

$$
\left(R_{H}^{-1}\right)_{\text {stat }}=\frac{M^{(0)}}{\pi \alpha}\left(1+\frac{M^{(1)}-4}{M^{(0)}} \frac{1}{\alpha}+\frac{M^{(2)}}{M^{(0)}} \frac{1}{\alpha^{2}}+\cdots\right) .
$$

Equivalently, to the same order in powers of $\alpha^{-1}$, the expression for $\left(R_{H}\right)_{\text {stat }}$ is given by

$$
\left(R_{H}\right)_{\mathrm{stat}}=\frac{\pi \alpha}{M^{(0)}}-\frac{\pi}{\left(M^{(0)}\right)^{2}}\left(M^{(1)}-4\right)+\frac{\pi}{M^{(0)}}\left[\left(\frac{M^{(1)}-4}{M^{(0)}}\right)^{2}-\frac{M^{(2)}}{M^{(0)}}\right] \frac{1}{\alpha}+\cdots
$$

Application of an inversion formula of [11] (see also [6]) to first solve for $\rho^{(0)^{\prime}}(b)$, for $b>0$ ) and then use of standard integration techniques to determine $\rho^{(0)}(b)$ shows that $(3.1)$ has as its solution

$$
\rho^{(0)}(b)=\frac{M^{(0)}}{\pi}\left[\frac{1}{4} \log \frac{\frac{1}{2}-\sqrt{\frac{1}{4}-b^{2}}}{\frac{1}{2}+\sqrt{\frac{1}{4}-b^{2}}}+\sqrt{\frac{1}{4}-b^{2}}\right]+\frac{k}{8} \log \frac{\frac{1}{2}-\sqrt{\frac{1}{4}-b^{2}}}{\frac{1}{2}+\sqrt{\frac{1}{4}-b^{2}}}+d
$$

where $k$ is an arbitrary constant and $d$ is the constant of integration. The solution for $\rho^{(0)}(b)$ on the symmetric interval $\left[-\frac{1}{2}, \frac{1}{2}\right]$ is then obtained by simply extending the result obtained on the interval $\left[0, \frac{1}{2}\right]$ as an even function.

The values of $k$ and $d$ are determined by the conditions $\rho^{(0)}(1 / 2)=0$ and $\int_{0}^{\frac{1}{2}} \rho^{(0)}(b) d b=1$. The value of $M^{(0)}$ is then obtained using (3.4). In practice, particularly in the determination of the higher order terms, it proves more convenient to determine the value of each $M^{(n)}$ by invoking the symmetry condition $\rho^{(n)^{\prime}}(0)=0$, rather than using the equations that define $M^{(n)}$ directly. 
By either approach, it can easily be shown that $k=-2 M^{(0)} / \pi$ and $d=0$, so that

$$
\rho^{(0)}(a)=-\int_{a}^{\frac{1}{2}} \rho^{(0)^{\prime}}(b) d b=M^{(0)} \sqrt{1 / 4-a^{2}} / \pi
$$

where

$$
1=\int_{0}^{\frac{1}{2}} \rho^{(0)}(a) d a=M^{(0)} / 16
$$

Thus, the zeroth order solution is given by

$$
\rho^{(0)}(a)=16 \sqrt{1 / 4-a^{2}} / \pi,
$$

and the zeroth order approximation to the stationary value of $R_{H}$ by

$$
\left(R_{H}^{(0)}\right)_{\mathrm{stat}}=\pi \alpha / 16
$$

Following the same procedure outlined above with the higher order terms yields the results (see [6] for details):

1. For the first approximation

$$
\rho^{(1)}(a)=-\frac{32}{\pi^{3}} \int_{a}^{\frac{1}{2}} \sqrt{\frac{1}{4}-b^{2}} \log \frac{\frac{1}{2}-b}{\frac{1}{2}+b} d b-\frac{32}{3 \pi^{3}} \sqrt{\frac{1}{4}-a^{2}}
$$

with

$$
M^{(1)}=4-128 /\left(3 \pi^{2}\right) \text {. }
$$

The first order approximation to the stationary value of $R_{H}$ is

$$
\left(R_{H}^{(1)}\right)_{\text {stat }}=\pi \alpha / 16+1 /(6 \pi)
$$

2. For the second approximation

$$
\begin{aligned}
\rho^{(2)}(a)= & -\frac{64}{\pi^{5}} \int_{0}^{\frac{1}{2}} \sqrt{\frac{1}{4}-t^{2}} \log \frac{\frac{1}{2}-t}{\frac{1}{2}+t} h(a, t) d t \\
& +\frac{64}{3 \pi^{5}} \int_{a}^{\frac{1}{2}} \sqrt{\frac{1}{4}-b^{2}} \log \frac{\frac{1}{2}-b}{\frac{1}{2}+b} d b-\frac{32}{9 \pi^{5}} \sqrt{\frac{1}{4}-a^{2}}
\end{aligned}
$$

where

$$
h(a, t) \equiv \int_{a}^{\frac{1}{2}} \log \left|\frac{b \sqrt{\frac{1}{4}-t^{2}}+t \sqrt{\frac{1}{4}-b^{2}}}{b \sqrt{\frac{1}{4}-t^{2}}-t \sqrt{\frac{1}{4}-b^{2}}}\right| d b
$$

with

$$
M^{(2)}=1+\frac{16}{\pi^{4}}\left(3-\frac{7}{2} \varsigma(3)\right)+\frac{160}{9 \pi^{4}}
$$


TABLE 1. Approximations to $R_{H}$

\begin{tabular}{|c|c|c|c|}
\hline$\alpha$ & Numerical & $R_{H}^{(1)}$ & $R_{H}^{(2)}$ \\
\hline 10.0 & 2.0168 & 2.0165 & 2.0168 \\
7.5 & 1.5259 & 1.5257 & 1.5260 \\
5.0 & 1.0351 & 1.0348 & 1.0353 \\
2.5 & 0.5444 & 0.5439 & 0.5448 \\
1.0 & 0.2496 & 0.2494 & 0.2517 \\
\hline
\end{tabular}

The second order approximation to the stationary value of $R_{H}$ is

$$
\left(R_{H}^{(2)}\right\rangle_{\mathrm{stat}}=\frac{\pi \alpha}{16}+\frac{1}{6 \pi}+\frac{\pi}{16}\left(\frac{3+\frac{7}{2} \zeta(3)}{\pi^{4}}-\frac{1}{16}\right) \frac{1}{\alpha}
$$

The function $\zeta$ appearing in the above equation is the Riemann $\zeta$-function and the coefficient of the term $1 / \alpha$ in the above expression for $\left(R_{H}^{(2)}\right)_{\text {stat }}$ is approximately 0.002256 to four significant figures.

\section{Discussion of results}

In this paper, the large- $\alpha$ approximation for the polarisability coefficient $R_{H}$ to the variational formulation of $[8,9]$ has been obtained. The values of $R_{H}^{(1)}$ and $R^{(2)}$, as given by (3.16) and (3.19) respectively, are given in Table 1 together with the values calculated by $[8,9]$ using a numerical Ritz procedure based on a variational modal technique for selected values of $\alpha$.

Agreement between the numerical and $R_{H}^{(2)}$ values is excellent for large values of $\alpha$. For smaller values of $\alpha$, it appears that $R_{H}^{(1)}$ provides a better representation. In fact this linear dependence of $R_{H}$ on $\alpha$ appears to reasonably represent the numerically obtained values for even quite small values of $\alpha$. The reason why this should be the case is currently under investigation, as is an attempt to determine the correct asymptotic representation of $R_{H}$ in the limiting case of $\alpha$ tending to zero.

The results summarised in Table 1 establish analytically that for rectangular apertures in thin shield walls the numerically generated results obtained previously for the variational formulation of $[8,9]$ are correct to better than four figure accuracy in the limiting case of the aspect ratio $\alpha$ large compared with unity.

\section{Acknowledgements}

The authors thank members of the Mathematics Department at RMIT for many helpful discussions, particularly J. Roger Blake (deceased), I. A. Peckham, T. Doukakaros and J. A. McPhee. 


\section{References}

[1] M. Abramowitz and I. A. Stegun, Handbook of mathematical functions with formulae, graphs and mathematical tables (Dover, N.Y., 1972).

[2] H. A. Bethe, "Lumped constants for small irises", Radiation Laboratory Report 43-22, 1943.

[3] H. A. Bethe, "Theory of diffraction by small holes", Phys. Rev. 66 (1944) 163-182.

[4] R. E. Collin, Field theory of guided waves (McGraw-Hill, N.Y., 1960).

[5] R. De Smedt and J. Van Bladel, "Magnetic polarisability of some small apertures", IEEE Trans. Antennas Propagat. AP-28 (1980) 703-707.

[6] G. F. Fitz-Gerald and N. A. McDonald, "An asymptotic solution of an integro-differentialequation arising in magnetic coupling through thin shield walls", RMIT, Department of Mathematics, Technical Report Series 9 (1987).

[7] I. S. Gradshteyn and I. M. Ryzhik, Tables of integrals series and products (Academic Press, N.Y., 1965).

[8] N. A. McDonald, "Electromagnetic coupling through small apertures", Ph.D. Thesis, University of Toronto, Canada, 1971. Also issued as Research Report 45, Dept. of Elec. Eng., Univ. of Toronto, 1971.

[9] N. A. McDonald "Electric and magnetic coupling through small apertures in shield walls of any thickness", IEEE Trans. Microwave Theory and Techniques MTT-20 (1972) 689695.

[10] N. A. McDonald, "Polynomial approximations for the transverse magnetic polarisabilities of some small apertures", IEEE Trans. Microwave Theory and Technigues MTT-35 (1987) 20-23.

[11] N. I. Muskhelishvili, Singular integral equations (Wolters-Noordhoff, Groningen, 1972). 